\title{
Are Multiple Descriptions Better Than One?
}

\author{
György Dán, Viktória Fodor, and Gunnar Karlsson \\ Department of Signals, Sensors and Systems, \\ KTH, Royal Institute of Technology \\ \{gyuri, viktoria, gk\}@imit.kth.se
}

\begin{abstract}
In this paper we compare three schemes for error recovery for real-time multimedia applications: media-dependent forward error correction (MD-FEC), proposed for real-time audio; media-independent forward error correction (MI-FEC), proposed for real-time video; and multiple description coding (MDC). We provide a detailed queueing analysis for these schemes considering bursty traffic sources, and combine results from information theory and queueing theory to analyze their performance. We conclude that MDC always performs better than MD-FEC, and that the average loss probability plays a key role in the choice of the optimal parameters. We also show that MDC outperforms MI-FEC if packet losses are highly correlated, like in the current Internet, and if the delay available for error control is low.
\end{abstract}

\section{Introduction}

Applications that require low loss probabilities in today's Internet have to employ some end-to-end mechanism to recover from packet losses. For interactive applications with strict delay constraints, the delay introduced by the applied schemes has to be low as well. Forward error correction (FEC) and the recently re-discovered multiple description coding (MDC) have been proposed for this purpose [1, 2, 3.

There are two main directions of FEC design to recover from packet losses. One solution, proposed by the IETF and implemented in Internet audio tools like Rat, is to add a redundant copy of the original packet to one of the subsequent packets. The redundant packet is highly compressed, so that the sound quality reconstructed from the redundant packet is low, but still better than when there is nothing to play out. Proposed ways to improve the performance of the scheme are to increase the time offset between the transmission of the original packet and the redundant one [4, and to send multiple redundant copies in subsequent packets [1]. The performance of this media-dependent FEC scheme (MD-FEC) has been evaluated via simulations in [5] and analytically in [6]. The results show that MD-FEC can be beneficial if the share of the total traffic implementing it is small. 
The other set of FEC solutions use block coding schemes based on algebraic coding, e.g. Reed-Solomon coding. The main idea behind these mediaindependent FEC schemes (MI-FEC) is to protect a block of $k$ packets of information with $c$ packets of redundancy. Thus the original $k$ packets can be reconstructed if out of $n=k+c$ packets at most $c$ packets are lost. MI-FEC has mainly been proposed for low-delay high-bitrate multimedia applications, such as real-time video, and has been extensively studied both via simulations and analytically [2,7]. The results are similar to those of MD-FEC: MI-FEC is efficient if only a small share of the streams uses it and the efficiency of MI-FEC increases as the block length, and thus the introduced delay, increases.

The traditional approach to networking, the separation of source and channel coding, was motivated by Shannon's separation theorem [8. It says that source coding and channel coding can be performed separately while maintaining optimality. However, Shannon's separation theorem assumes that the available delay is unlimited, which is not true for real-time applications. MDC addresses the problem of joint source and channel coding. Originally it was designed for the transmission of multiple descriptions of a single source over independent channels. If only one of the descriptions is received, it is used for reconstruction with a certain accuracy. If more than one descriptions are received, then the information from the other descriptions can be used to enhance the accuracy. It has been rediscovered recently for use in packet switched networks [3]: instead of using separate channels, one can put the different encodings into different packets and send them with a time offset, similarly to the case of MD-FEC. In general the amount of information sent over the separate channels can be different, however in single-path packet networks it can be shown that balanced MDC, i.e. the one sending the same amount of information in all packets is optimal [3].

In this paper we compare the performance of the two FEC schemes and MDC via combining results from information theory and queueing theory. The optimal selection of source and redundancy rates for real-time audio was investigated before in [1] using the Gilbert channel model. A similar approach was followed in [9] with respect to MPEG coded video. The performance of MDC was compared to that of single description coding in the context of content delivery networks via simulations in [10. The authors considered an average packet loss rate of $5 \%$ and concluded that MDC can reduce the distortion by as much as 20 to $40 \%$. The performance of MI-FEC and MDC with $50 \%$ redundancy was compared in [11] using the Gilbert channel model. As the authors used a fixed redundancy rate for FEC, the comparison gave advantage to MDC by allowing it to adjust to the channel characteristics.

The rest of the paper is organized as follows. In Section 2 we describe the queueing model used to calculate the conditional loss probability of packets. In Section 3 we introduce the distortion rate bounds for FEC and MDC. In Section 4 we compare the performance of MD-FEC and MDC to be used for low-bitrate multimedia streams. In Section 5 we compare the performance of MI-FEC and MDC suitable for high-bitrate multimedia streams. We conclude our work in Section 6 . 


\section{Analysis of the Packet Loss Process}

In this section we first give an overview of past work on the modeling of the packet loss correlation, then we turn to the calculation of the conditional packet loss probability. We will use the conditional loss probability and the probability of $j$ losses in a block of $n$ packets in the remainder of the paper to calculate the average distortion of the various error correction schemes under different network conditions.

Schulzrinne et al. derived the consecutive loss probability for the $N * I P P / D / 1 / K$ queue and showed that it can be orders of magnitude higher than the loss probability [12. The conditional loss probability was analyzed under the assumption of exponential interarrival time and service time distribution in [6]. The authors considered a single flow and a packet spacing smaller than the queue length. They also presented a model with exponential interarrival time and general service time distribution and gave simple lower and upper bounds on the consecutive loss probability.

\subsection{Model Description}

We model the network as a single queue, representing the bottleneck in the transmission path 13, 14, with Erlang-r distributed packet sizes having average transmission time $1 / \mu$. Using the Erlang-r distribution makes the mathematical analysis simple, while it can be used to get approximate results on the performance of FEC by matching the mean and variance of the packet size distribution to measurements [15]. Packets arrive to the system from two sources, a Markovmodulated Poisson process (MMPP) and a Poisson process, representing the tagged source and the background traffic respectively. The packets are stored in a buffer that can host up to K packets, and are served according to a FIFO policy.

It is known that compressed media, e.g. VBR video, exhibits a self-similar nature [16. Ryu and Elwalid [17] showed, however, that short term correlations have dominant influence on the network performance under realistic scenarios of buffer sizes for real-time traffic. Thus the MMPP may be a practical model to derive approximate results for the queueing behavior of compressed media, especially in the case of small buffer sizes.

The assumption of the Poisson background traffic is partly justified by recent results indicating that Internet traffic can be approximated by a non-stationary Poisson process [18. According to measurements, the change free intervals are well above $150 \mathrm{~ms}$, the ITU's G.114 recommendation for end-to-end delay for real-time applications. These empirical results are consistent with recent theoretical results [19].

We assume that the sources feeding the system are independent. The MMPP is described by the infinitesimal generator matrix $Q$ with elements $r_{l m}$ and the arrival rate matrix $\Lambda=\operatorname{diag}\left\{\lambda_{1}, \ldots, \lambda_{L}\right\}$, where $\lambda_{l}$ is the average arrival rate while the underlying Markov chain is in state $l[20$. The Poisson process modeling the background traffic has average arrival rate $\lambda$. The superposition of the two 
sources can be described by a single MMPP with arrival rate matrix $\hat{\Lambda}=\Lambda \oplus \lambda=$ $\Lambda+\lambda I$, and infinitesimal generator $\hat{Q}=Q$, where $\oplus$ is the Kronecker sum. Each packet in the queue corresponds to $r$ exponential stages of service, and the state space of the queue is $\{0, \ldots, r K\} \times\{1, \ldots, L\}$.

\subsection{Conditional Packet Loss}

Our purpose is to calculate the probability of the event that an arbitrary packet from the tagged source arrives in state $i$ of the system and the $n^{\text {th }}$ next packet arrives in state $j$ of the system $P_{i, j}(n), n \geq 0,0 \leq i, j \leq r K$. We define the probability $P_{j \mid i}^{m \mid l}(n)$ (and $\left.\bar{P}_{j \mid i}^{m \mid l}(n)\right), 0 \leq i, j \leq r K, 1 \leq l, m \leq L, n \geq 0(n \geq 1)$ as the probability of the event that the $n^{\text {th }}$ next packet from the tagged source is generated while the MMPP is in state $m$ and arrives in state $j$ of the system, given that the remaining number of exponential stages in the system is $i$ just before the arrival of the first (next) packet from the tagged stream (background traffic) and the MMPP is in state $l$. As the first packet is arbitrary,

$$
P_{i, j}(n)=\sum_{l=1}^{L} \Pi(i, l) \sum_{m=1}^{L} P_{j \mid i}^{m \mid l}(n) .
$$

$\Pi(i, l)$, the steady state distribution of the exponential stages in the queue as seen by an arriving packet can be calculated as

$$
\Pi(i, l)=\frac{\pi(i, l) \lambda_{l}}{\sum_{l=1}^{L} \lambda_{l} \sum_{i=0}^{r K} \pi(i, l)},
$$

where $\pi(i, l)$ is the steady state distribution of the $M M P P / E_{r} / 1 / K$ queue [15].

The probabilities $P_{j \mid i}^{m \mid l}(n)$ can be derived according to the following recursion. The recursion is initiated for $n=0$ with the following relations

$$
P_{j \mid i}^{m \mid l}(0)=\left\{\begin{array}{ll}
1 & \text { if } j=i \text { and } m=l \\
0 & \text { otherwise }
\end{array} .\right.
$$

Using the notation $p_{u}=\frac{\lambda_{u}}{\lambda_{u}+\lambda}$ and $\bar{p}_{u}=\frac{\lambda}{\lambda_{u}+\lambda}$, for $n \geq 1$ the following equations hold.

$$
\begin{gathered}
P_{j \mid i}^{m \mid l}(n)=\sum_{u=1}^{L} \sum_{k=0}^{i+r} Q_{i+r}^{u \mid l}(k)\left\{p_{u} P_{j \mid i+r-k}^{m \mid u}(n-1)+\bar{p}_{u} \bar{P}_{j \mid i+r-k}^{m \mid u}(n-1)\right\} \quad 0 \leq i \leq r(K-1), \\
P_{j \mid i}^{m \mid l}(n)=\sum_{u=1}^{L} \sum_{k=0}^{i} Q_{i}^{u \mid l}(k)\left\{p_{u} P_{j \mid i-k}^{m \mid u}(n-1)+\bar{p}_{u} \bar{P}_{j \mid i-k}^{m \mid u}(n-1)\right\} \quad r(K-1)<i, \quad(5) \\
\bar{P}_{j \mid i}^{m \mid l}(n)=\sum_{u=1}^{L} \sum_{k=0}^{i+r} Q_{i+r}^{u \mid l}(k)\left\{p_{u} P_{j \mid i+r-k}^{m \mid u}(n)+\bar{p}_{u} \bar{P}_{j \mid i+r-k}^{m \mid u}(n)\right\} \quad 0 \leq i \leq r(K-1), \quad(6) \\
\bar{P}_{j \mid i}^{m \mid l}(n)=\sum_{u=1}^{L} \sum_{k=0}^{i} Q_{i}^{u \mid l}(k)\left\{p_{u} P_{j \mid i-k}^{m \mid u}(n)+\bar{p}_{u} \bar{P}_{j \mid i-k}^{m \mid u}(n)\right\} \quad r(K-1)<i .
\end{gathered}
$$


$Q_{i}^{u \mid l}(k)$ denotes the joint probability that the next arrival will be in state $u$ of the MMPP and that $k$ exponential stages out of $i$ will be completed before the next arrival from the joint arrival process, given that the last arrival was in state $l$ of the MMPP. A way to calculate $Q_{i}^{u \mid l}(k)$ is shown in [15. The procedure of computing $P_{j \mid i}^{m \mid l}(n)$ follows that of computing $P(j, n)$ in [15].

Let us now define two sets of states, $\alpha$ and $\omega$ as the set of states of the queue where arriving packets can enter the system and where arriving packets are discarded respectively. Then $\alpha=\{0 \ldots r(K-1)\}$ and $\omega=\{r(K-1)+1 \ldots r K\}$. Using these notations the probability that a packet arriving to the system from the tagged source is not lost and the $n^{\text {th }}$ next packet from the tagged source is lost is given as $p_{\alpha \omega}(n)=\sum_{i \in \alpha} \sum_{j \in \omega} P_{i, j}(n)$. The probabilities $p_{\alpha \alpha}(n), p_{\omega \alpha}(n)$ and $p_{\omega \omega}(n)$ can be defined in a similar way. Throughout the paper we refer to $p_{\omega \mid \omega}(n)$ as the consecutive loss probability for $n=1$ and as the conditional loss probability for $n \geq 1$.

\subsection{Losses in a Block}

We use the method presented in [15] to calculate the probability of $j$ losses in a block of $n$ packets $P(j, n), n \geq 1,0 \leq j \leq n$ for the described network model. Based on the probabilities $P(j, n)$, one can calculate the probability that a packet is uncorrectable by MI-FEC, given the parameters $n$ and $k$, as $P_{u c}(n, k)=$ $\sum_{j=n-k+1}^{n} j P(j, n) / n$.

\section{Distortion-Rate Bounds for FEC and MDC}

When compressing signals, there is always a tradeoff between the size and the accuracy of the representation. This tradeoff, the bounds on achievable rates and distortions, is represented by the distortion-rate and the rate-distortion functions, which depend on the source characteristics and the distortion measure. We consider a memoryless Gaussian source with unit variance and use the mean squared error distortion measure, which is the most common distortion measure. The distortion-rate function for a Gaussian source with unit variance and the squared distortion measure is given as

$$
D(R)=2^{-2 R}
$$

where $R$ is the code rate and $D(R)$ is the distortion 3 . In the following we discuss the distortion-rate characteristics of the three considered error control schemes.

In the case of MD-FEC the first and the subsequent $\nu-1$ (redundant) descriptions are encoded independently of each other, and thus, if any of them is received, its distortion is given by (8). We denote the rate allocated to the primary encoding with $R_{1}^{M D F}$ and the rate allocated to the $k^{\text {th }}$ (redundant) copy with $R_{k}^{M D F}$. The redundancy ratio introduced by the MD-FEC is then $\beta=\sum_{k=2}^{\nu} R_{k}^{M D F} / R_{1}^{M D F}$, and the total rate of the source is $R^{M D F}=R_{1}^{M D F}(1+\beta)$. If both the primary and 
some redundant encodings are received, the redundant encodings can not be used to reduce the distortion.

In the case of MI-FEC each packet corresponds to an encoding of rate $R_{1}^{M I F}$. The packets compose blocks, and redundancy is added to the block of packets. If we denote the ratio of redundancy with $\beta=(n-k) / k$, then the total rate is given as $R^{M I F}=R_{1}^{M I F}(1+\beta)$. If the number of lost packets in a block is no more than $c$ then the original packets can be reconstructed and the distortion of the individual packets is given by (8).

In the case of MDC we denote the total source rate with $R^{M D C}$ and consider the balanced two channel case. We denote the rate allocated to individual descriptions with $R_{1}^{M D C}=R^{M D C} / 2$. The distortion when both descriptions are received, called the central distortion, is denoted by $D_{0}^{M D C}$ and the distortion if only one of the descriptions is received, called the side distortion, is denoted with $D_{1}^{M D C}$. The distortion rate bounds for the 2-channel MDC are [3]:

$$
\begin{aligned}
D_{1}^{M D C} & \geq 2^{-2 R^{M D C} / 2} \\
D_{0}^{M D C}\left(D_{1}^{M D C}\right) & \geq 2^{-2 R^{M D C}} \gamma\left(R^{M D C}, D_{1}^{M D C}\right),
\end{aligned}
$$

where $\gamma\left(R^{M D C}, D_{1}^{M D C}\right)=1$ if $2 D_{1}^{M D C}>1+D_{0}^{M D C}$ and otherwise

$$
\gamma\left(R^{M D C}, D_{1}^{M D C}\right)=\left\{1-\left\{\left(1-D_{1}^{M D C}\right)-\sqrt{\left(D_{1}^{M D C}\right)^{2}-2^{-2 R^{M D C}}}\right\}^{2}\right\}^{-1} .
$$

Equation (10) shows that if the side distortion is small then the central distortion is higher than the distortion rate minimum (8). For a primer on rate-distortion theory and multiple description coding see 3 .

In general the source rate $R$ (bits per symbol) and the bitrate of the stream $\mathrm{C}$ (bits per second) are related to each other through the symbol rate $\mathrm{S}$ (symbols per second) as $C=S \times R$. For example, a video sequence with a small image size can reach a higher source rate $R$ at the same bitrate as another sequence with a big image size. For this reason in the following analysis we will distinguish between these two parameters.

\section{MD-FEC Versus MDC}

In this section we consider a source that has an available source rate $R_{a}$ and can use either MD-FEC or MDC for error control due to the low delay bounds and low bitrate. For simplicity, we will consider the case of $\nu=2$. Using the notations introduced in Section 3 the mean distortion bound of the MD-FEC scheme can be calculated as the weighted sum of the distortions of the cases when both descriptions are received, when only one of them is received or when none of them is received

$$
D^{M D F}(\beta)=\left(p_{\alpha \alpha}(n)+p_{\alpha \omega}(n)\right) D\left(R_{1}^{M D F}\right)+p_{\omega \alpha}(n) D\left(R_{2}^{M D F}\right)+p_{\omega \omega}(n),
$$

where the loss probabilities were defined in Section 2.2. For a redundancy ratio $\beta$ the rates of the individual descriptions are $R_{1}^{M D F}=R_{a} /(1+\beta)$ and $R_{2}^{M D F}=$ $R_{a} \beta /(1+\beta)$. The level of redundancy that minimizes (12) is the solution $\beta_{*}^{M D F}$ 
of $\frac{\partial D^{M D F}(\beta)}{\partial \beta}=0$. Using the distortion rate function introduced in Section 3 the solution is

$$
\beta_{*}^{M D F}=\frac{\ln \left(2^{2 R_{a}}\right)-\ln \left(\frac{p_{\alpha \alpha}(n)+p_{\omega \alpha}(n)}{p_{\omega \alpha}(n)}\right)}{\ln \left(2^{2 R_{a}}\right)+\ln \left(\frac{p_{\alpha \alpha}(n)+p_{\omega \alpha}(n)}{p_{\omega \alpha}(n)}\right)}=\frac{\ln \left(2^{2 R_{a}}\right)-\ln \left(\frac{1-p_{\omega}(n)}{p_{\omega}(n)\left(1-p_{\omega \mid \omega}(n)\right)}\right)}{\ln \left(2^{2 R_{a}}\right)+\ln \left(\frac{1-p_{\omega}(n)}{p_{\omega}(n)\left(1-p_{\omega \mid \omega}(n)\right)}\right)} .
$$

We denote the minimal mean distortion by $D_{*}^{M D F}$. Based on (13) the optimal ratio of redundancy $\beta_{*}^{M D C}$ is positive only if

$$
p_{\omega}>\left\{1+D\left(R_{a}\right)^{-1}\left(1-p_{\omega \mid \omega}(n)\right)\right\}^{-1}
$$

Thus for a given source rate it is not beneficial to use MD-FEC below this average loss probability. Furthermore, if $p_{\omega \mid \omega}(n)$ is not close to 1 , the minimum value of $p_{\omega}$ is dominated by $D\left(R_{a}\right)^{-1}$. In general, for any distortion rate function of the form $D(R)=a b^{-c R}$ the limit given in (14) is $p_{\omega}>\left\{1+a D\left(R_{a}\right)^{-1}(1-\right.$ $\left.\left.p_{\omega \mid \omega}(n)\right)\right\}^{-1}$.

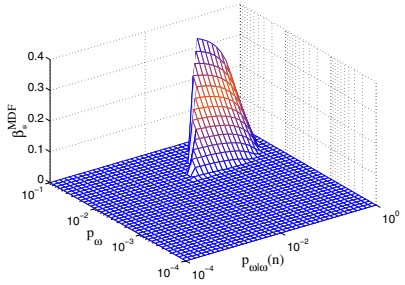

Fig. 1. Optimal rate of redundancy vs. $p_{\omega}$ and $p_{\omega \mid \omega}(n)$ for $D\left(R_{a}\right)=0.01$

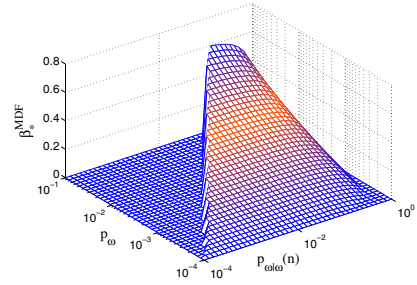

Fig. 2. Optimal rate of redundancy vs. $p_{\omega}$ and $p_{\omega \mid \omega}(n)$ for $D\left(R_{a}\right)=10^{-4}$

By substituting $\beta_{*}^{M D F}$ given by (13) into (12) it can be shown analytically that increasing $n$ decreases the average distortion whenever $p_{\omega}$ satisfies (14) since $p_{\omega \mid \omega}(n)$ is a decreasing function of $n$. This result is in accordance with the empirical observations presented in 4. If, however, $p_{\omega} \leq 1 /\left(1+D\left(R_{a}\right)^{-1}\right)$ then increasing $n$ does not decrease the distortion. The value $\beta_{*}^{M D F}$ that minimizes the average distortion at a given average loss probability and conditional loss probability is shown in Fig. 1 and 2 for distortions $D\left(R_{a}\right)=10^{-2}$ and $D\left(R_{a}\right)=$ $10^{-4}$ respectively. Comparing the figures we see that the rate of the source and the average loss probability have a big influence on whether or not MD-FEC should be used.

Now we consider the case of the balanced MDC scheme with two descriptions. The total available rate is $R_{a}$, and thus the rates of the individual descriptions are $R_{1}^{M D C}=R_{2}^{M D C}=R_{a} / 2$. The mean distortion bound can be calculated as $D^{M D C}\left(D_{1}^{M D C}\right)=p_{\alpha \alpha}(n) D_{0}^{M D C}\left(D_{1}^{M D C}\right)+\left(p_{\alpha \omega}(n)+p_{\omega \alpha}(n)\right) D_{1}^{M D C}+p_{\omega \omega}(n)$. 


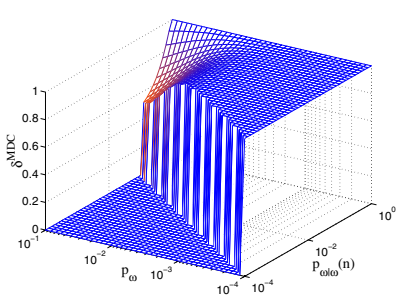

Fig. 3. MDC gain vs. $p_{\omega}$ and $p_{\omega \mid \omega}(n)$ for $D\left(R_{a}\right)=0.01$

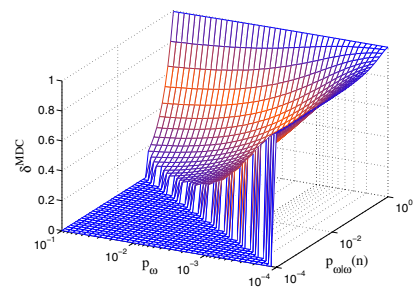

Fig. 4. MDC gain vs. $p_{\omega}$ and $p_{\omega \mid \omega}(n)$ for $D\left(R_{a}\right)=10^{-4}$

The optimal combination of central and side distortions can be found by setting the first derivative with regard to $D_{1}^{M D C}$ of (15) to zero [11]. The solution is given by

$$
\frac{\partial D_{0}^{M D C}\left(D_{1}^{M D C}\right)}{\partial D_{1}^{M D C}}=-\frac{p_{\alpha \omega}(n)+p_{\omega \alpha}(n)}{p_{\alpha \alpha}(n)}=-\frac{p_{\omega}\left(1-p_{\omega \mid \omega}(n)\right)}{1+p_{\omega \mid \omega}(n) p_{\omega}-2 p_{\omega}} .
$$

We denote the minimal mean distortion by $D_{*}^{M D C}$. To compare the performance of MD-FEC and MDC we define $\delta_{M D F}^{M D C}=D_{*}^{M D C} / D_{*}^{M D F}$, the ratio of the mean distortion bounds. Similarly we define $\delta^{M D C}=D_{*}^{M D C} /\left(p_{\alpha} D\left(R_{a}\right)+p_{\omega}\right)$, the ratio of the mean distortion bound with MDC to the bound without any error control. Figs. 3 and 4 show $\delta^{M D C}$ as a function of the average loss probability and the conditional loss probability for distortions $D\left(R_{a}\right)=10^{-2}$ and $D\left(R_{a}\right)=10^{-4}$. They show that the available source rate, the average loss probability and the conditional loss probability together determine the potential of MDC to decrease the mean distortion. Figs. 5 and 6 show $\delta_{M D F}^{M D C}$ as a function of the average loss probability and the conditional loss probability for distortions $D\left(R_{a}\right)=10^{-2}$ and $D\left(R_{a}\right)=10^{-4}$. They show that MDC reduces the mean distortion more efficiently than MD-FEC under all circumstances. The difference, however, is not large, up to a factor of 25\%. Comparing these figures to Figs. 1 and 2 , we see that MDC outperforms MD-FEC primarily in those regions of average loss and conditional loss probability where $\beta_{*}^{M D F}>0$. It follows that error correction with MDC becomes practically useless below the loss probability given in (14).

The value of the parameters $p_{\omega}$ and $p_{\omega \mid \omega}(n)$ used in the evaluation presented above can be taken from measured traces, simulations or mathematical models. In the remainder of the section we use the model presented in Section 2 to evaluate the effects of various traffic and network parameters on the loss correlation. We consider a scenario, possibly VoIP transmission, where the average arrival intensity is $50 / \mathrm{sec}$ and the packets have a constant size of 160 bytes, thus the corresponding bitrate would be $64 \mathrm{kbps}$. The interarrival time between packets from the tagged source is exponentially distributed. This assumption can be justified by the fact that packets from a flow cross several routers before arriving to the bottleneck router [6]. The background traffic is modeled by a Poisson process, packet sizes are exponentially distributed with an average packet length of 454 

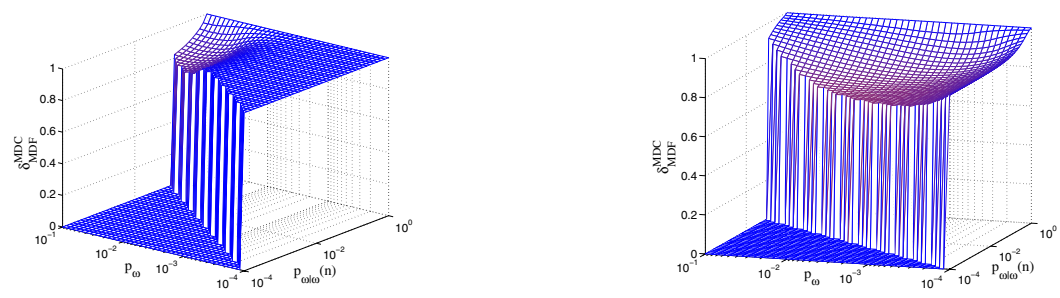

Fig. 5. $\delta_{M D F}^{M D C}$ vs. $p_{\omega}$ and $p_{\omega \mid \omega}(n)$ for $D\left(R_{a}\right)=0.01$

Fig. 6. $\delta_{M D F}^{M D C}$ vs. $p_{\omega}$ and $p_{\omega \mid \omega}(n)$ for $D\left(R_{a}\right)=10^{-4}$
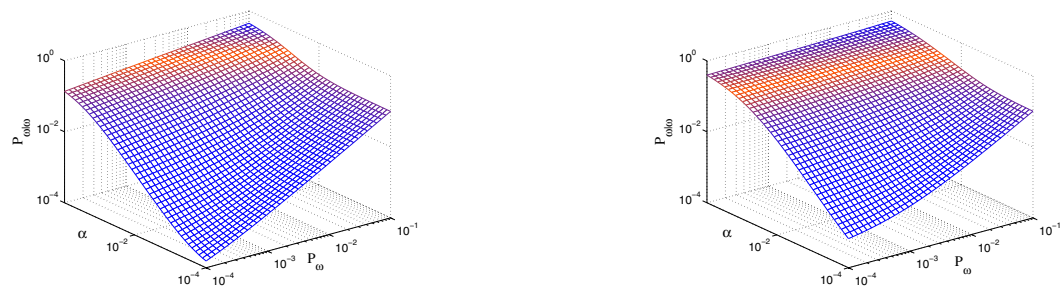

Fig. 7. $p_{\omega \mid \omega}(1)$ vs. average loss probability and $\alpha$ for $\mathrm{K}=5$

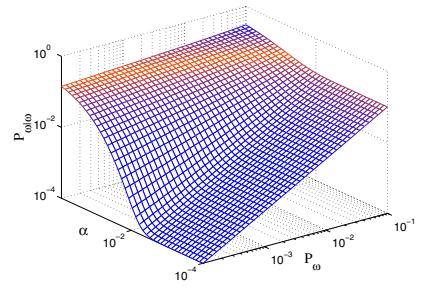

Fig. 9. $p_{\omega \mid \omega}(4)$ vs. average loss probability and $\alpha$ for $\mathrm{K}=20$

Fig. 8. $p_{\omega \mid \omega}(1)$ vs. average loss probability and $\alpha$ for $\mathrm{K}=20$

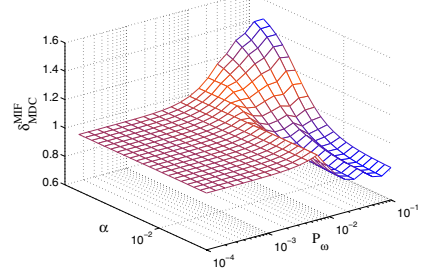

Fig. 10. $\delta_{M D C}^{M I F}$ vs. average loss probability and $\alpha$ for $K=5, n=10$ and $D\left(R_{a}\right)=10^{-2}$

bytes. This value closely matches the average packet size of the Internet traffic traces shown in [21. We denote by $\alpha$ the probability that an arriving packet belongs to the tagged source, and consider values between $10^{-4}$ and 1 . These values correspond to a background traffic between 0 and $226 \mathrm{Mbps}$. Depending on the value of $\alpha$ the packet size distribution of the aggregate traffic will differ from exponential. We approximate the resulting packet size distribution by means of the Erlang-r distribution. For a given value of $\alpha$ we change the link capacity to achieve different average loss probabilities, between $10^{-4}$ and $10^{-1}$. Figs. 7 and 8 show $p_{\omega \mid \omega}(1)$ as a function of the average loss probability and $\alpha$ for a queue capacity of $K=5$ and $K=20$ respectively. The figures show that at high loss rates the conditional loss probability reaches the average loss probability faster 
than at low loss rates. Fig. 9] shows $p_{\omega \mid \omega}(4)$ (a spacing of 4 packets corresponds to a delay of $80 \mathrm{~ms}$ ) as a function of $p_{\omega}$ and $\alpha$ for queue capacity $K=20$. The figure shows that the lower the value of $\alpha$ the higher the decrease of $p_{\omega \mid \omega}$ due to the increased delay. This has been observed in measurements on the public Internet 22 .

\section{MI-FEC Versus MDC}

In the following section we consider an arbitrary packet, part of a high bitrate multimedia stream and evaluate how MI-FEC and MDC can protect the data against losses. Using the notations of Section 3 the mean distortion bound of MI-FEC for given block length $n$, ratio of redundancy $\beta$ and available rate $R_{a}$ is

$$
D^{M I F}(n, k)=\left(1-P_{u c}(n, k)\right) D\left(\frac{R_{a}}{1+\beta}\right)+P_{u c}(n, k),
$$

where $P_{u c}(n, k)$ was defined in Section 2.3. We consider the case that the block length $n$ is given, and if the source wants to increase the ratio of redundancy then it has to decrease its source rate. Thus in order to minimize the mean distortion we select the value of $k$ that minimizes (17), and denote the minimum by $D_{*}^{M I F}$.

The mean distortion bound of the two-channel MDC and the optimal combination of side and central distortions for a given loss process have been shown in (15) and (16) in Section 4. We choose the spacing between the two descriptions to be $n-1$ packets, where $n$ is the block length of MI-FEC. This way the same delay is introduced by the two schemes.

In the following we consider a scenario, where the tagged source is a 3 -state Markov modulated Poisson process with arrival intensities $\lambda_{1}=116 / \mathrm{s}, \lambda_{2}=$ $274 / s, \lambda_{3}=931 / s$ and transition rates $r_{12}=0.12594, r_{21}=0.25, r_{23}=1.97, r_{32}=$ 2. These values were derived from an MPEG-4 coded trace by matching the average bitrates of the I, $\mathrm{P}$ and $\mathrm{B}$ frames and the corresponding transition intensities. We use the same model for the MD coded video as there are no models proposed for it. The packet size of the tagged source is fixed to 188 bytes, as for the transport stream in the MPEG-2 standard [23. Based on these parameters the bitrate of the source is $540 \mathrm{kbps}$. The assumptions on the background traffic, the aggregate packet size distribution and the definition of $\alpha$ are the same as in Section 4. We change the value of $\alpha$ between $10^{-3}$ and 1 , these values correspond to a background traffic of 0 to $162 \mathrm{Mbps}$. We change the link capacity to achieve different average loss probabilities between $10^{-4}$ and $10^{-1}$.

To compare the performance of MI-FEC and MDC we define $\delta_{M D C}^{M I F}=$ $D_{*}^{M I F} / D_{*}^{M D C}$, the ratio of the mean distortion bounds. Fig. 10 shows $\delta_{M D C}^{M I F}$ as a function of the average loss probability and $\alpha$ for a source with distortion $D\left(R_{a}\right)=10^{-2}$, buffer capacity $K=5$ and block length $n=10$ ( $\approx 30$ ms delay). The figure shows that below the loss probability given in (14) neither MI-FEC nor MDC can decrease the distortion. Above this loss probability MDC outperforms MI-FEC if $\alpha$ is high, e.g. losses are correlated (see Fig. 7). The reason for this phenomenon is that the redundant information needed to recover a lost 
packet with MI-FEC is carried in subsequent packets. Thus if losses occur in bursts, a high ratio of redundancy is needed to recover from losses. In the case of MDC however, descriptions are placed as far away as possible and so the loss process as seen by MDC is less bursty. Measurements performed on the Internet 22 confirm that losses occur independently above spacing $n>20$. The "valley" shapes parallel to the $\alpha$ axis are due to the fact that $\beta$ for MI-FEC can not be adjusted continuously. Figure 11 shows the same scenario for a block length of $n=20$ ( $\approx 60$ ms delay). Comparing the figures shows an increasing performance

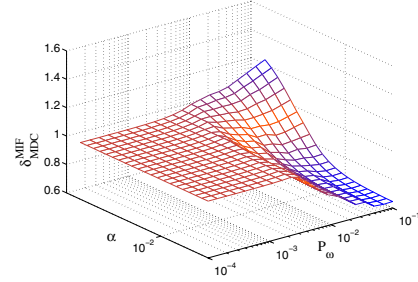

Fig. 11. $\delta_{M D C}^{M I F}$ vs. average loss probability and $\alpha$ for $K=5, n=20$ and $D\left(R_{a}\right)=10^{-2}$

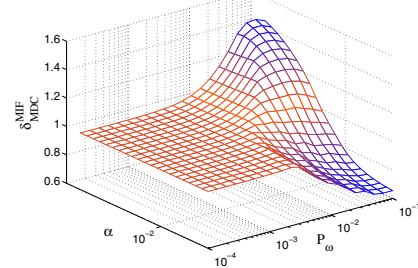

Fig. 13. $\delta_{M D C}^{M I F}$ vs. average loss probability and $\alpha$ for $K=20, n=20$ and $D\left(R_{a}\right)=10^{-2}$

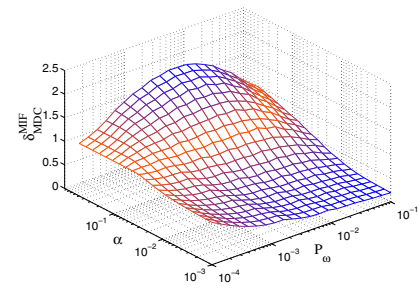

Fig. 15. $\delta_{M D C}^{M I F}$ vs. average loss probability and $\alpha$ for $K=20, n=20$ and $D\left(R_{a}\right)=10^{-4}$

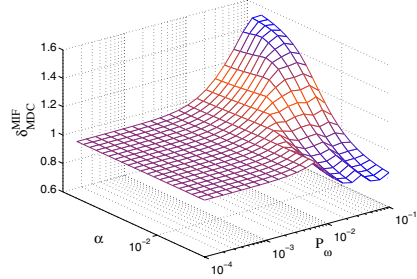

Fig. 12. $\delta_{M D C}^{M I F}$ vs. average loss probability and $\alpha$ for $K=20, n=10$ and $D\left(R_{a}\right)=10^{-2}$

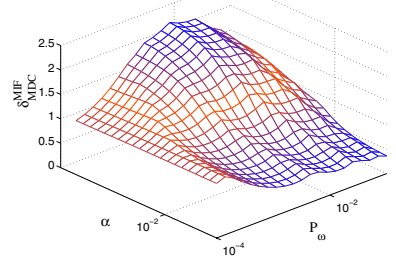

Fig. 14. $\delta_{M D C}^{M I F}$ vs. average loss probability and $\alpha$ for $K=20, n=10$ and $D\left(R_{a}\right)=10^{-4}$

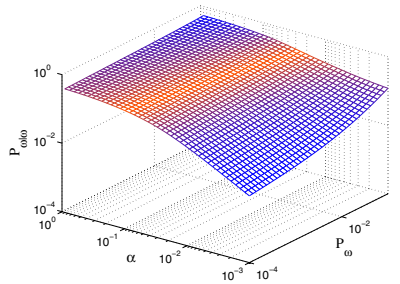

Fig. 16. $p_{\omega \mid \omega}(1)$ vs. average loss probability and $\alpha$ for $K=20$, bursty traffic 
of MI-FEC as the block length increases. In fact, as the block length increases, MI-FEC can correct longer bursts at the same redundancy ratio. By increasing the buffer size, $M$ to 20 , we see that MDC performs better over a wider range of parameters $p_{\omega}$ and $\alpha$ as shown in Figs. 12 and 13. This is due to the higher correlation between losses as an effect of the increased queue length (see Figs. 7 and 8). Figs. 14, 15 show results for the same scenarios for distortion $D\left(R_{a}\right)=10^{-4}$.

Recent measurements on the Internet [22, 24] show that the average loss probability is around $1 \%$ while the consecutive loss probability around $25 \%$. Considering $K=20$ and $D\left(R_{a}\right)=10^{-4}$, these parameters correspond to $\alpha=0.0305$ (Fig. 16), and thus for $n=5,10$ and 20 we get $\delta_{M D C}^{M I F}=2.32,1.5$ and 0.91 respectively (Figs. 14 and 15). The choice of an average loss probability of $5 \%$ and consecutive loss probability $20 \%$ as in [10] corresponds to $\alpha=0.016$ and leads to $\delta_{M D C}^{M I F}=1.45,0.96$ and 0.59 for $n=5,10$ and 20 respectively. These examples suggest that for short delays MDC performs better than MI-FEC on today's Internet.

\section{Conclusions and Discussion}

In this paper we presented an analytical evaluation of the potential of forward error correction and multiple description coding to recover from losses under various network conditions. We compared media-dependent FEC to MDC and concluded that MDC gives better performance under all circumstances. The comparison is based on the loss probabilities, thus it is independent of the traffic parameters. We showed that for a big class of distortion-rate models, the optimal ratio of redundancy is mainly determined by the average packet loss probability. We proposed a queueing model to evaluate the loss process of bursty traffic and used the model to compare the performance of media independent FEC and MDC with two descriptions. We showed that MDC gives better performance if losses are correlated and the delay available for error control is low. In today's Internet losses are correlated and network end-to-end delays are large, thus leaving short delays for error control. Hence we conclude that even MD coding with two descriptions could improve the quality of real-time multimedia communications.

Based on the results presented in this paper we believe that multiple description coding is an appealing error control solution for delay sensitive traffic in an environment with correlated packet losses like the Internet.

\section{References}

1. J. C. Bolot, S. Fosse-Parisis, and D. Towsley, "Adaptive FEC-based error control for Internet telephony," in Proc. of IEEE INFOCOM, pp. 1453-1460, 1999.

2. E. Biersack, "Performance evaluation of forward error correction in ATM networks," in Proc. of ACM SIGCOMM, pp. 248-257, August 1992.

3. V. K. Goyal, "Multiple description coding: Compression meets the network," IEEE Signal Processing Mag., September 2001. 
4. I. Kouvelas, O. Hodson, W. Hardman, and J. Crowcroft, "Redundancy control in real-time Internet audio conferencing," in Proc. of International Workshop on Audio-Visual Services Over Packet Networks, September 1997.

5. M. Podolsky, C. Romer, and S. McCanne, "Simulation of FEC-based error control for packet audio on the Internet," in Proc. of IEEE INFOCOM, pp. 505-515, 1998.

6. E. Altman, C. Barakat, and V. Ramos, "Queuing analysis of simple FEC schemes over IP," Computer Networks, vol. 39, pp. 185-206, June 2002.

7. K. Kawahara, K. Kumazoe, T. Takine, and Y. Oie, "Forward error correction in ATM networks: An analysis of cell loss distribution in a block," in Proc. of IEEE INFOCOM, pp. 1150-1159, June 1994.

8. C. E. Shannon, "A mathematical theory of communication," Bell Syst. Tech. J., pp. 379-423, 1948.

9. P. Frossard and O. Verscheure, "Joint source/FEC rate selection for qualityoptimal MPEG-2 video delivery," IEEE Trans. Image Processing, vol. 10, no. 12, pp. 1815-1825, 2001.

10. J. Apostolopoulos, T. Wong, W. Tan, and S. Wee, "On multiple description streaming with content delivery networks," in Proc. of IEEE INFOCOM, pp. 1736-1745, 2002.

11. M. Y. Kim and B. W. Kleijn, "Rate-distortion comparisons between FEC and MDC based on Gilbert channel model," in Proc. of IEEE ICON, pp. 495-500, 2003.

12. H. Schulzrinne, J. Kurose, and D. Towsley, "Loss correlation for queues with bursty input streams," in Proc. of IEEE ICC, pp. 219-224, 1992.

13. J. C. Bolot, "End-to-end packet delay and loss behavior in the Internet," in Proc. of ACM SIGCOMM, pp. 289-298, September 1993.

14. O. J. Boxma, "Sojourn times in cyclic queues - the influence of the slowest server," Computer Performance and Reliability, pp. 13-24, 1988.

15. G. Dán, V. Fodor, and G. Karlsson, "Packet size distribution: an aside?," in Proc. of QoS-IP'05, pp. 75-87, February 2005.

16. J. Beran, R. Sherman, M. Taqqu, and W. Willinger, "Long-range dependence in variable-bit-rate video traffic," IEEE Trans. Commun., vol. 43, no. 2/3/4, pp. 1566-1579, 1995.

17. B. Ryu and A. Elwalid, "The importance of long-range dependence of VBR video traffic in ATM traffic engineering: Myths and realities," in Proc. of ACM SIGCOMM, pp. 3-14, 1996.

18. T. Karagiannis, M. Molle, and M. Faloutsos, "A nonstationary poisson view of Internet traffic," in Proc. of IEEE INFOCOM, pp. 1-12, March 2004.

19. R. Gaigalas and I. Kaj, "Convergence of scaled renewal processes and a packet arrival model," Journal of the Bernoulli Society for Mathematical Statistics and Probability, vol. 9, no. 4, pp. 671-703, 2003.

20. W. Fischer and K. Meier-Hellstern, "The markov-modulated poisson process MMPP cookbook," Performance Evaluation, vol. 18, no. 2, pp. 149-171, 1992.

21. Sprint IP Monitoring Project, "http://ipmon.sprint.com/."

22. M. Yajnik, S. Moon, J. Kurose, and D. Towsley, "Meaurement and modelling of the temporal dependence in packet loss," in IEEE INFOCOM, pp. 345-352, 1999.

23. D. Hoffman and G. Fernando, "RTP payload format for MPEG1/MPEG2 video," RFC 2250, Jan. 1998.

24. I. Marsh, F. Li, and G. Karlsson, "Wide area measurements of VoIP quality," in Proc. of Quality of Future Internet Services, pp. 63-72, October 2003. 